\title{
Are Indian Cricket Fast Bowlers at Risk of Injury? A 3D Biomechanical Investigation
}

\author{
Sai Aditya Krishna, ${ }^{1,}{ }^{*}$ Thiagarajan Alwar, ${ }^{1}$ Anees Sayed, ${ }^{1}$ Gnanavel MB, ${ }^{1}$ and Arumugam Sivaraman ${ }^{1}$ \\ ${ }^{1}$ Centre for Sports Science (CSS), Sri Ramachandra University, Chennai, Tamil Nadu, India \\ "Corresponding author: Sai Aditya Krishna, Sreenivas, 19, 12th Cross St., Indira Nagar, Adyar, Chennai - 600020, India. Tel: +91-9940119000, E-mail: tablasai@gmail.com
}

Received 2017 July 12; Accepted 2018 January 23.

\begin{abstract}
Background: Cricket is the most commonly played and followed sport in India. Modern day cricket demands a high level of consistent performance from the fast bowler, who carries the highest risk for injury. 3D motion analysis can be used to prevent injuries by identifying incorrect techniques. Researchers have identified key biomechanical variables as predictors of injury in fast bowlers. Very little such information is available with respect to Indian cricket fast bowlers despite the huge popularity of the sport here. Objectives: To identify areas of injury risk in Indian fast bowlers with respect to key biomechanical variables, using 3D motion analysis.

Methods: 22 male State level fast bowlers between ages 18-30 years underwent 3D motion analysis in an indoor cricket biomechanics testing facility. Bowling action was captured with a set of 3D cameras (250 fps) and 2D video cameras (125 fps). Data processing \& analysis was done with proprietary software using a standard model. Key biomechanical variables including delivery Stride Length (SL), Lateral Trunk Flexion at Ball Release (LTF at BR) and Knee Flexion at Front Foot Contact (KF at FFC) were measured. Peak Vertical Ground Reaction Forces (pVGRF) were recorded simultaneously using force plates. Descriptive statistical analysis of results was done.

Results: A large proportion of bowlers were at risk of injuries (100\% had less than optimum SL, 41\% had high LTF at BR, $45 \%$ had high pVGRF and $45 \%$ had low KF at FFC). Bowling action type was found to be ideal or acceptable in $77 \%$.

Conclusions: Indian fast bowlers, in spite of possessing less injurious bowling action types, are vulnerable to injury with respect to key biomechanical variables. This emphasises role of 3D motion analysis in Indian state level fast bowlers for early identification of injurious techniques and could have implications on coaching of fast bowlers in India.
\end{abstract}

Keywords: Cricket, Fast Bowling, Cricket Injuries, Biomechanics

\section{Background}

Modern day cricket demands a high level of consistent performance from the fast bowler, who carries the highest injury risk (1). Bowling technique has been identified as a crucial factor for an effective and injury free fast bowling career (2). Implementation of technology such as video analysis and 3 - Dimensional motion analysis have enabled bowlers to enhance their fast bowling technique to improve performance and prevent injuries, by early identification of faulty techniques. 3D Motion analysis is now the gold - standard tool to assess the kinematics of fast bowling action. Many researchers have identified key biomechanical variables that can predict injury risk and have even established cut - off values for risk of injury. However, most of these studies have been done in the African, Caucasian and Australian populations. Despite the large popularity of the sport in India, there is a paucity of literature on the biomechanical analysis of fast bowling action, mostly due to non - availability of resources.

\section{Objectives}

This study aimed to perform 3D motion analysis of the bowling action of Indian cricket fast bowlers and identify the injury risks with respect to key biomechanical variables.

\section{Methods}

This cross sectional study was conducted in the Centre for Sports Science (CSS) at Sri Ramachandra University, Chennai, between September 2015 and May 2016. Approval from the host institution's Ethics Committee was obtained. 22 male fast bowlers from state \& top division 
level cricket teams under the Tamil Nadu Cricket Association (TNCA) who came to CSS for training camps were included in the study.

\subsection{Selection Criteria}

Inclusion criteria were: State or $1^{\text {st }} / 2^{\text {nd }}$ division level male cricket fast bowlers between the ages 18 - 30 years.

Exclusion criteria were: Current injuries at the time of the study or within the past 6 months which required rest from playing or training for more than one week.

\subsection{Bowling Testing Procedure}

The subjects were asked to bowl wearing suitable running shoes without spikes in the indoor facility, which has an artificial turf (Uni - Turf, Sports Surfaces Ltd., UK). Testing was scheduled at a fixed time of the day under ambient natural light. Written informed consent was obtained. A set of 32 Retro - reflective markers were affixed to the participant's skin over anatomical landmarks using a pre - adhesive spray and double sided tape, based on Plug In Gait model (Plug - In Gait Marker Set, Vicon Peak, Oxford, UK) (Figure 1). Anthropometric measurements were taken as per the Plug - in gait recommendations using a joint Anthropometer. 12 Vicon MX T20 - S cameras (@250 fps) (Vicon, Oxford Metrics, Oxford, UK) were used to collect the kinematic data. Two Bonita 2D video cameras (@125 fps) placed behind the bowler at a height of 3 meters and to the side of the bowling crease 2 meters away from the pitch were used to record the bowling motion. Ground reaction forces were recorded simultaneously using two force foot plates (AMTI BP600900-1000 Advanced Mechanical Technology Inc., USA) which were embedded beneath the surface at the bowling crease and covered with the same artificial turf for seamless camouflage. Ball speed was recorded using a hand held radar speed gun (Pocket Radar Ball Coach, Pro - Level Speed Training Tool and Radar Gun) from the batsman's end. Static \& Dynamic (Wand) calibration of the setup as per Vicon's guidelines was done (Figure 2). Subjects carried out a self - directed warm - up and bowled one over (consisting of six deliveries). They were instructed to bowl a good line and length (measured using a standard method (3)) at match pace.

\subsection{Data Processing}

Data processing and reconstruction was done by the same biomechanist for all the bowlers using the Plug - in Gait pipeline on Vicon Nexus (Version 1.8.5) software. Data was filtered using a Woltring filter. The best delivery based on completeness of data capture (both markers and force plates) from each bowler was selected for analysis (Figure 3).
LTF at BR was measured as peak sideward flexion of the trunk segment relative to the pelvis segment in the frontal plane at two frames ahead of visual separation of ball from hand. Front knee angle was measured at FFC and at maximum knee flexion as the angle between the femur segment and the tibia segment. Front knee action was calculated based on the classification by Portus (3). The distance between the two malleoli markers at FFC was measured as SL and expressed as a percentile ratio of body height. pVGRF was measured as the peak force in the $\mathrm{Z}$ axis from the force plate data at the frame of FFC (Figure 4). Bowling action typing was done according to traditional classification (2, 3).

\subsection{Statistical Analysis}

Descriptive Statistical Analysis of the data was done using IBM SPSS ${ }^{\circledR}$ (Version 23.0). The means of the variables were compared to the mean values from previous literature and injury risk was determined based on established cut - off values from previous literature.

\section{Results}

Demographics of the study population is shown in Table 1. Descriptive statistical analysis of the kinetic \& kinematic parameters is shown in Table 2. Mean values of the kinetic \& kinematic parameters were compared to reference values from previous literature. Mean pVGRF, LTF at BR, Maximum FK Flexion were similar to reference values from previous studies (2-6). However, mean KF at FFC was lower than reference values (6). Mean SL was greatly lower than reference values and all bowlers had less than optimum SL $(2,5)$. Further analysis was done by comparing the kinematic data with cut - off values for injury risk from previous literature (Figure 5). 40.9\% had high LTF at BR, 45.4\% had high pVGRF \& low KF at FFC. Bowling action type was found to be ideal to acceptable in $77 \% .23 \%$ of bowlers had "Mixed" type of bowling action (Figure 6). Only 32\% of bowlers used the Flexor - Extender type of Front Knee Action (Figure 7).

\section{Discussion}

This is the first study which describes 3D biomechanical analysis of Indian cricket fast bowlers from an injury risk perspective. The results of this study demonstrate several key areas of injury risk which can be corrected by targeted interventions such as technique modifications and corrective exercises \& thus reduce the injury risk.

High pVGRF when not adequately dissipated, is a known direct stressor of the contralateral pars inter - articularis leading to lumbar spine injury $(5,7)$. Fast bowlers 

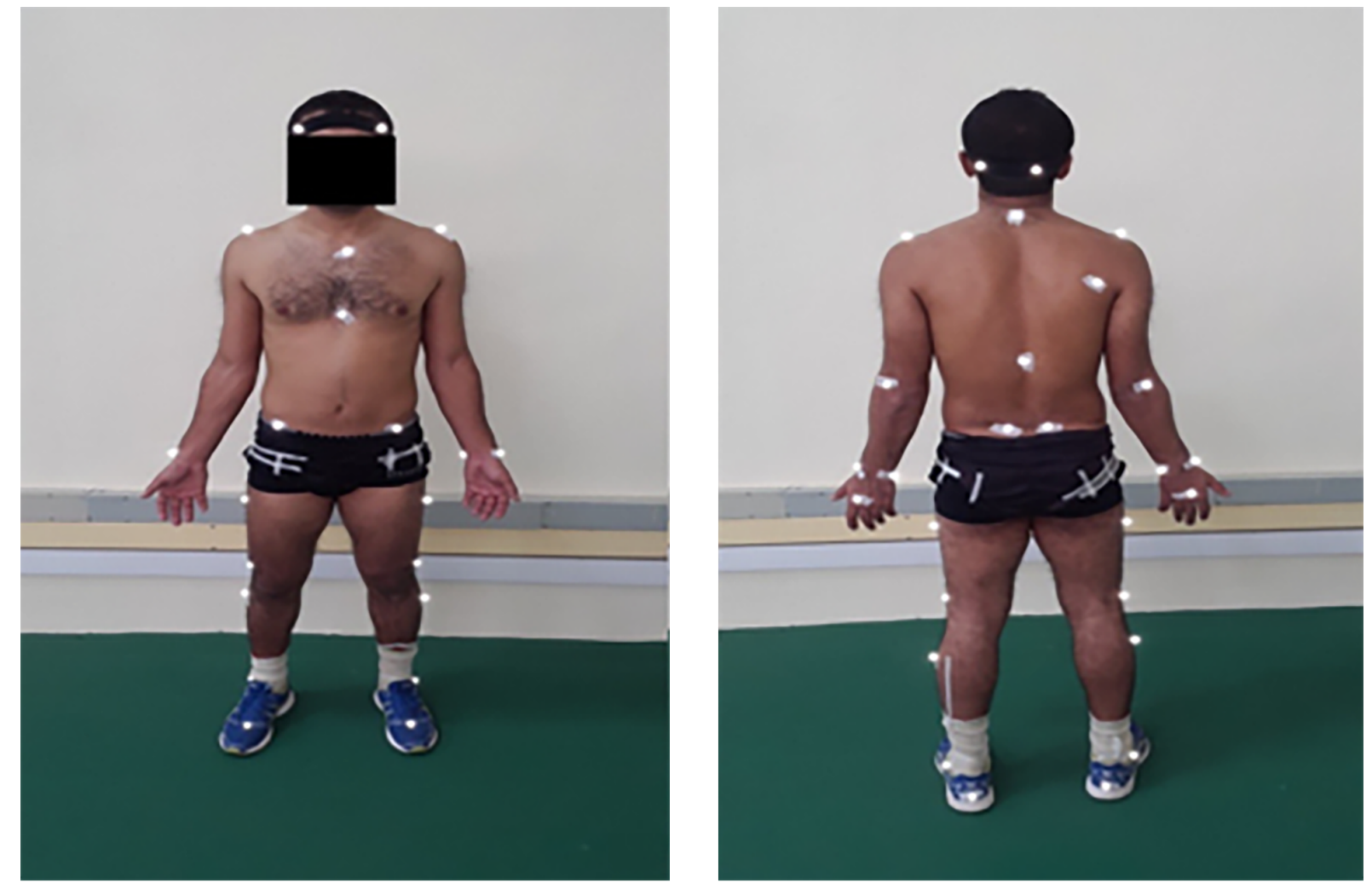

Figure 1. Bowler with Plug - in Gait Marker Setup

\begin{tabular}{lcccc}
\hline Table 1. Demographic Data of the Population & & & \\
\hline & Min. & Max. & Mean & Std. Dev. \\
\hline Age & 18.00 & 30.00 & 21.45 & 179.60 \\
Height $(\mathbf{C m})$ & 170.50 & 191.00 & 7.65 \\
Weight $(\mathbf{K g})$ & 61.30 & 130.00 & 77.56 & 13.93 \\
\hline
\end{tabular}

\begin{tabular}{lcc}
\hline Table 2. Kinetic and Kinematic Data & & \\
\hline Parameter & Mean & Std. Dev. \\
\hline pVGRF $(\mathbf{x}$ BW) & 6.50 & 1.61 \\
\hline Max KF $\left(^{\circ}\right)$ & 40.19 & 20.16 \\
\hline KF at FFC $\left(^{\circ}\right)$ & 13.57 & 7.89 \\
\hline LTF at BR $\left(^{\circ}\right)$ & 39.65 & 7.21 \\
\hline SL $(\%$ BH $)$ & 64.49 & 4.93 \\
\hline Top Speed at Good Length $(\mathbf{k m} / \mathbf{h r})$ & 120.20 & 6.49 \\
\hline
\end{tabular}

generate large forces at both feet during the delivery and up to 9 times the body weight at FFC $(2,8)$. It was previously thought the bowlers could utilise a higher pVGRF to generate faster bowling speeds. However, a recent study by
King and colleagues found no correlation between pVGRF \& bowling speed thus proving that a high pVGRF is unnecessary for fast bowling (9). $45 \%$ of bowlers in our study had a pVGRF higher than the mean pVGRF of injured bowlers in Portus' study indicating injury risk.

Increased lateral trunk flexion also acts as a stressor on the contralateral lumbar spine $(4,10,11)$. In our study, bowlers had a mean lateral trunk flexion of $39.65^{\circ} \pm 7.21$ which is very near the injury risk cut off value. $41 \%$ of bowlers had LTF higher than $40^{\circ}$ placing them at risk of lumbar spine injury.

The mixed - action bowler tends to have more hyperextension, lateral flexion and greater rotation which greatly loads the lumbar spine. In our study, we found that only $23 \%$ of bowlers had a "Mixed" type of action and 50\% of 


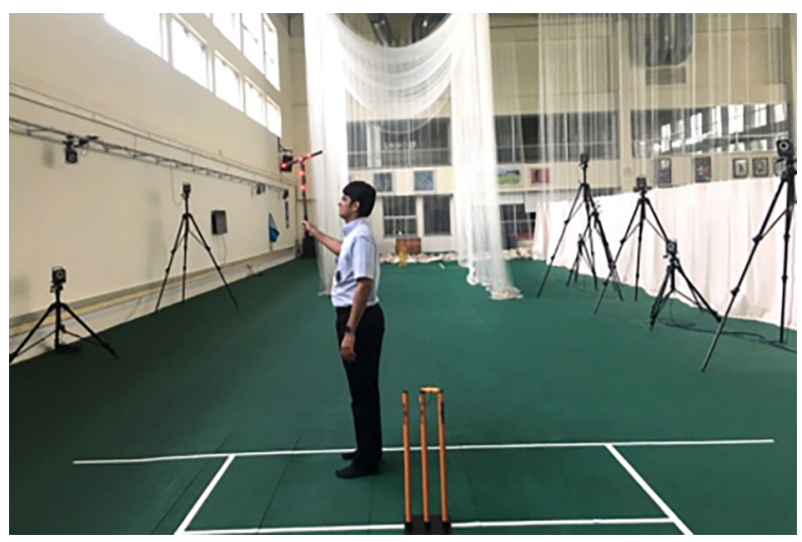

Figure 2. Biomechanics Laboratory - Dynamic Calibration

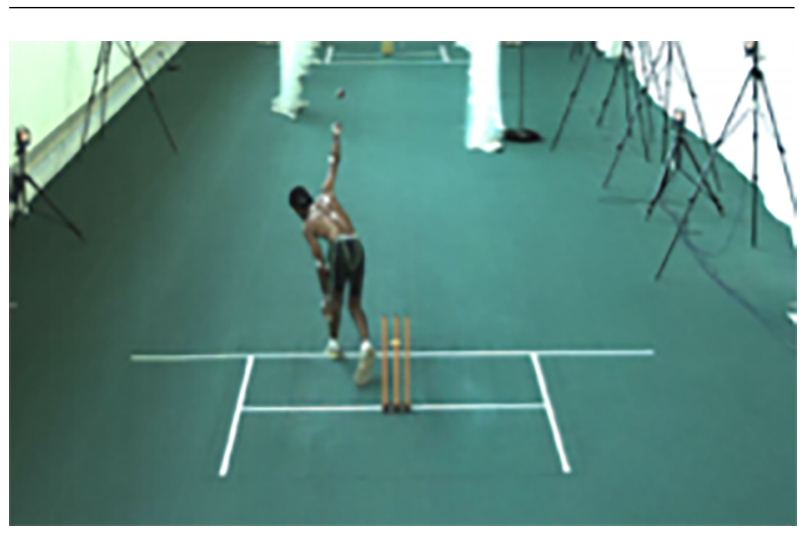

Figure 3. 2D Video Analysis

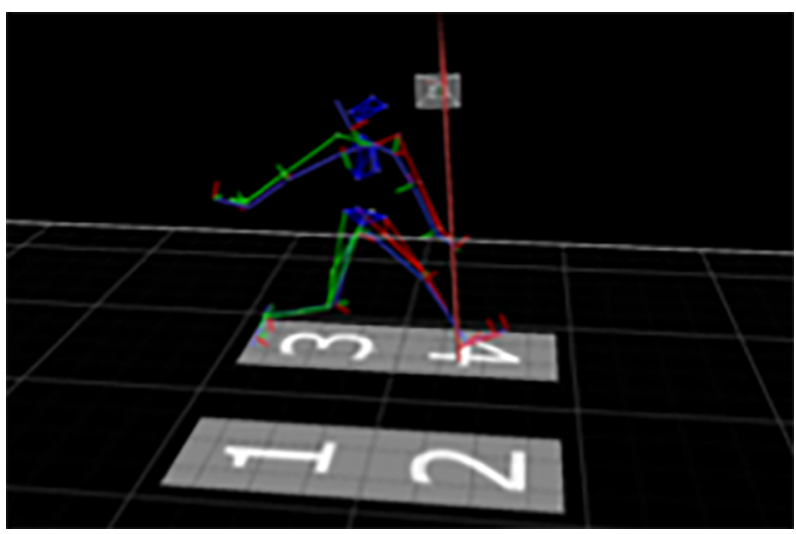

Figure 4. 3D Reconstructed Image for Motion Analysis

bowlers had the "Semi Open" type of action. "Side On" action incidence was low which was in agreement with other studies. Thus it can be presumed that a good number of In- dian fast bowlers are using a less injurious type of action, though this has to be confirmed by a larger scale cross sectional study.

The front leg acts as a shock absorber to attenuate the ground reaction forces upon front foot impact. The front knee flexion angle has strong correlations with both the lumbar spine rotation and lateral flexion and therefore has an important effect on lumbar spine loading (11). A more extended front knee during the front foot contact phase has been associated with spondylolysis development $(3,5)$. This implies that more knee flexion on FFC leads to better GRF absorption. However, this reduces the ball release height leading to slower ball speeds. Thus, the 'optimum' front leg action is considered to be one that lands extended or slightly flexed, followed by a period of flexion to absorb ground reaction forces, before vigorously extending up to the point of ball i.e. Flexor - Extender type $(3,12)$.

In our study, bowlers had a mean knee flexion at FFC of $13.5^{\circ} \pm 8^{\circ}$. In a prospective study by Crewe et al., bowlers without lumbar spine stress injury, had a mean KF at FFC of $14.2 \pm 6.3^{\circ}(4) .55 \%$ of our bowlers had a knee angle at FFC higher than $14.2^{\circ}$. This suggests that Indian fast bowlers land with the knee in more extension compared to the other fast bowlers and hence, are at a higher risk of injury. Only $32 \%$ of bowlers used the ideal Flexor-Extender type of front knee action, which is efficient and injury free.

Authors have suggested an optimal delivery stride length of $75 \%$ - $85 \%$ of standing height (5). A shorter stride length inhibits the ability of the bowler to master a side on position, thus increasing the potential for shoulder counter rotation causing lumbar spine injuries $(5,13)$. As stride length increases, there is a greater amount of plantar flexion increasing the risk of posterior ankle impingement (14). In our study, the mean stride length of $64.5 \%$ $\mathrm{BH} \pm 4.93$ was much lower than the Western population. All bowlers in this study had a shorter than optimal stride length. Thus Indian fast bowlers are possibly at a comparatively higher risk of posterior ankle impingement as well as lumbar spine injuries. However, we acknowledge the fact that stride length could have been influenced by the nature of indoor lab testing due to factors such as run up length, approach speed, bowling effort, inability to use spiked bowling shoes, artificial turf etc.

In summary, a comparatively higher risk of lumbar spine, knee and ankle injuries was found in this study. Coaching interventions which are aimed at optimising the type of bowling action and technique corrections that help bowlers utilise the Flexor - Extender type of front knee action would help in significantly reducing the potential risk of these injuries. 


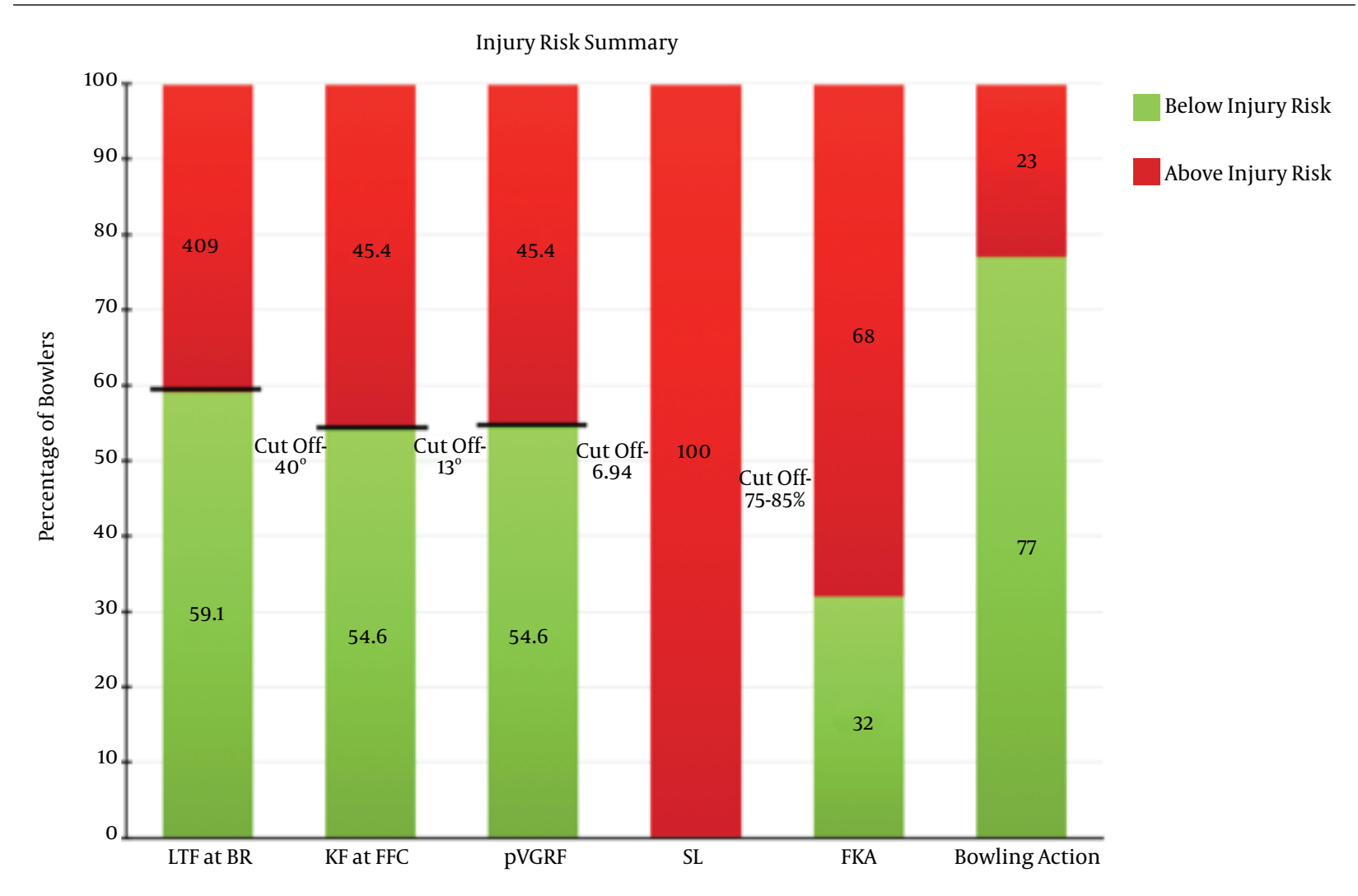

Figure 5. Injury Risk Summary

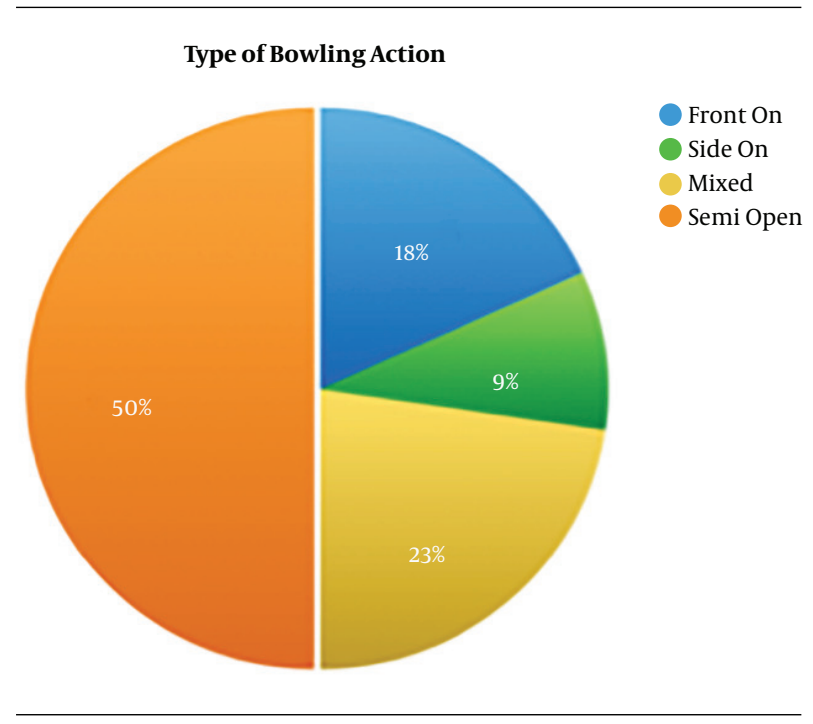

Figure 6. Bowling Action Classification

\subsection{Conclusions}

Despite possessing an acceptable type of bowling action, a high proportion of Indian fast bowlers are vulner-

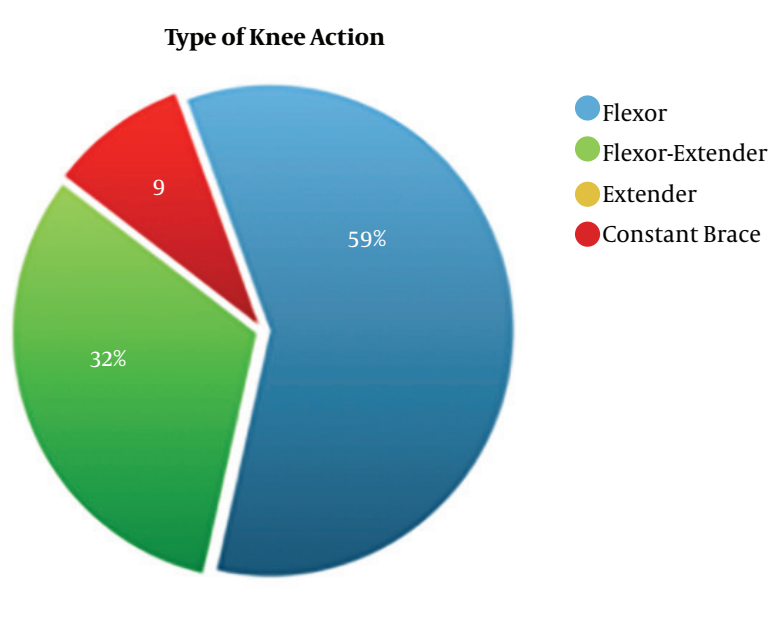

Figure 7. Front Knee Action Classification

able to injury with respect to key biomechanical variables. This emphasises role of 3D motion analysis for early identification of injurious techniques and could thus have implications on coaching of fast bowlers in India. 


\section{References}

1. Stretch RA. Cricket injuries: a longitudinal study of the nature of injuries to South African cricketers. BrJ Sports Med.2003;37(3):250-3. discussion 253. [PubMed: 12782551]. [PubMed Central: PMC1724632].

2. Bartlett RM, Stockill NP, Elliott BC, Burnett AF. The biomechanics of fast bowling in men's cricket: a review. J Sports Sci. 1996;14(5):403-24. doi: 10.1080/02640419608727727. [PubMed: 8941911].

3. Portus MR, Mason BR, Elliott BC, Pfitzner MC, Done RP. Cricket. Sport Biomech. 2004;3(2):263-84. doi: 10.1080/14763140408522845.

4. Crewe H, Campbell A, Elliott B, Alderson J. Lumbo-pelvic loading during fast bowling in adolescent cricketers: the influence of bowling speed and technique. J Sports Sci. 2013;31(10):1082-90. doi: 10.1080/02640414.2012.762601. [PubMed: 23363419].

5. Foster D, John D, Elliott B, Ackland T, Fitch K. Back injuries to fast bowlers in cricket: a prospective study. Br J Sports Med.1989;23(3):1504. [PubMed: 2620228]. [PubMed Central: PMC1478681].

6. Worthington P, Ranson C, King M, Burnett A, Shine K. The effect of individualised coaching interventions on elite young fast bowler's technique, School of Sport and Exercise Science, Loughborough University, UK 1 UK Athletics Chief Physiotherapist Loughborough, UK 2 School of Exercise. Biomedical \& Heal. 2008:3-6.

7. Elliott BC. Back injuries and the fast bowler in cricket. $J$ Sports Sci. 2000;18(12):983-91. doi: 10.1080/026404100446784. [PubMed: 11138988].

8. Hurrion PD, Dyson R, Hale T. Simultaneous measurement of back and front foot ground reaction forces during the same delivery stride of the fast-medium bowler. J Sports Sci. 2000;18(12):993-7. doi: 10.1080/026404100446793. [PubMed: 11138989].

9. King MA, Worthington PJ, Ranson CA. Does maximising ball speed in cricket fast bowling necessitate higher ground reaction forces? J Sports Sci. 2016;34(8):707-12. doi: 10.1080/02640414.2015.1069375. [PubMed: 26186222].

10. Ranson CA, Burnett AF, King M, Patel N, O'Sullivan PB. The relationship between bowling action classification and three-dimensional lower trunk motion in fast bowlers in cricket.J Sports Sci. 2008;26(3):267-76 doi: 10.1080/02640410701501671. [PubMed: 17926175].

11. Ferdinands R, Stuelcken M, Sinclair P, Greene A, Smith R, editors. Lumbar kinematics and kinetics of young Australian fast bowlers. ISBS Conference Proceedings Archive. 2010.

12. Glazier PS, Wheat JS. An integrated approach to the biomechanics and motor control of cricket fast bowling techniques. Sports Med. 2014;44(1):25-36. doi: 10.1007/s40279-013-0098-x. [PubMed: 24065337].

13. Elliott BC, Hardcastle PH, Burnett AE, Foster DH. The influence of fast bowling and physical factors on radiologic features in high performance young fast bowlers. Sports Med Train Rehabil. 1992;3(2):113-30. doi: $10.1080 / 15438629209517008$

14. Spratford W, Hicks A. Increased delivery stride length places greater loads on the ankle joint in elite male cricket fast bowlers. J Sports Sci. 2014;32(12):1101-9. doi: 10.1080/02640414.2014.886130. [PubMed: 24576107]. 